\title{
Asymptotic Generalized Fermat's Last Theorem over Number Fields
}

\author{
Yasemin Kara, Ekin Ozman
}

\begin{abstract}
Recent work of Freitas and Siksek showed that an asymptotic version of Fermat's Last Theorem holds for many totally real fields. Later this result was extended by Deconinck to generalized Fermat equations of the form $A x^{p}+B y^{p}+C z^{p}=0$, where $A, B, C$ are odd integers belonging to a totally real field. Another extension was given by Şengün and Siksek. They showed that the Fermat equation holds asymptotically for imaginary quadratic number fields satisfying usual conjectures about modularity.

In this work, combining their techniques we extend their results about the generalized Fermat equations to imaginary quadratic fields. More specifically we prove that the asymptotic generalized Fermat's Last Theorem holds for many quadratic imaginary number fields.
\end{abstract}

\section{Introduction}

Let $K$ be a number field and $\mathcal{O}_{K}$ be its ring of integers. In [4], Freitas and Siksek proved the asymptotic Fermat's Last Theorem over a totally real field $K$. That is, they showed that there is a constant $B_{K}$ such that for any prime $p>B_{K}$, the only solutions to the Fermat equation $a^{p}+b^{p}+c^{p}=$ 0 where $a, b, c \in \mathcal{O}_{K}$ are the trivial ones satisfying $a b c=0$. They gave an algorithmically testable criterion which, in case satisfied by $K$, implies the asymptotic Fermat's Last Theorem over $K$ and they proved that their criterion is satisfied by real quadratic fields $K=\mathbb{Q}(\sqrt{d})$ for a subset of $d \geq 2$ with density $5 / 6$ among the other squarefree positive integers.

Then, Deconinck [3] extended the results of Freitas and Siksek [4] to the generalized Fermat equation of the form $A a^{p}+B b^{p}+C c^{p}=0$ where $A, B, C$ are odd integers belonging to a totally real field. In a recent paper [8], Şengün and Siksek proved the asymptotic Fermat's Last Theorem for any number field $K$ by assuming two deep but standard conjectures form the Langlands programme. They also deduced that the asymptotic Fermat's Last Theorem holds for imaginary quadratic fields $\mathbb{Q} \sqrt{-d}$ with $-d \equiv 2,3(\bmod 4)$ squarefree.

The purpose of this paper is to extend the results of [8] to the generalized Fermat equation of the form $A a^{p}+B b^{p}+C c^{p}=0$ where $A, B, C$ are odd integers belonging to any number field $K$. In addition, we prove three density results, similar to the ones given in [8], 3] and [4].

\section{Our results}

Before we state our results, we set up some notations. Let $K$ be a number field and $\mathcal{O}_{K}$ be its ring of intergers. Let $A, B, C$ be non-zero elements of $\mathcal{O}_{K}$, and $p$ be a prime. We refer the equation

$$
A a^{p}+B b^{p}+C c^{p}=0, \quad a, b, c \in \mathcal{O}_{K}
$$


as the generalized Fermat equation over $K$ with coefficients $A, B, C$ and exponent $p$. A solution $(a, b, c)$ is called trivial if $a b c=0$, otherwise non-trivial. We set up the following notation throughout the paper.

- $R:=\operatorname{Rad}(A B C)=\prod_{\mathfrak{q} \text { prime in } \mathcal{O}_{K}}^{\mathfrak{q} \mid A B C} \mathfrak{q}$

- $S:=\left\{\mathfrak{P}: \mathfrak{P}\right.$ is a prime ideal of $\mathcal{O}_{K}$ such that $\left.\mathfrak{P} \mid 2 R\right\}$,

- $T:=\left\{\mathfrak{P}: \mathfrak{P}\right.$ is a prime ideal of $\mathcal{O}_{K}$ above 2$\}$,

- $U:=\{\mathfrak{P} \in T: f(\mathfrak{P} / 2)=1\}$.

Our main theorem depends on two deep but standard conjectures, which will be explained in the following section. These conjectures are the same ones assumed in [8]. Their assumption is necessary since the analogues of Modularity Theorem have not proved yet in general.

Main Theorem. Let $K$ be a number field satisfying Conjectures 2.1 and 2.2. Let $A, B, C \in \mathcal{O}_{K}$ and suppose that $A, B, C$ are odd, in the sense that if $\mathfrak{P}$ is a prime of $\mathcal{O}_{K}$ lying over 2, then $\mathfrak{P} \nmid A B C$. Write $\mathcal{O}_{S}^{*}$ for the set of $S$-units of $K$. Suppose that for every solution $(\lambda, \mu)$ to the $S$-unit equation

$$
\lambda+\mu=1, \quad \lambda, \mu \in \mathcal{O}_{S}^{*},
$$

there is some $\mathfrak{P} \in U$ that satisfies $\max \left\{\left|v_{\mathfrak{P}}(\lambda)\right|,\left|v_{\mathfrak{P}}(\mu)\right|\right\} \leq 4 v_{\mathfrak{P}}(2)$. Then there is a constant $\mathcal{B}=\mathcal{B}(K, A, B, C)$ such that the generalized Fermat equation with exponent $p$ and coefficients $A, B, C$ (given in equation 1.1) does not have non-trivial solutions with $p>\mathcal{B}$. We refer to this as "the asymptotic generalized Fermat's Last Theorem holds for K."

Also, we prove three density results.

Density Theorem 1. Let $K=\mathbb{Q}(\sqrt{-d})$ be an imaginary quadratic field where $d$ is a squarefree positive integer such that $-d \equiv 2,3(\bmod 4)$. Let $q \geq 29$ be a prime such that $q \equiv 5(\bmod 8)$ and $\left(\frac{-d}{q}\right)=-1$. Assume Conjectures 2.1 and 2.2. Then there exists a constant depending on $K$ and $q$, namely $B_{K, q}$, such that for all $p>B_{K, q}$ the Fermat equation $x^{p}+y^{p}+q^{r} z^{p}=0$ doesn't have any non-trivial solutions.

Density Theorem 2. Let $K=\mathbb{Q}(\sqrt{-d})$ be an imaginary quadratic field where $d$ is a squarefree positive integer such that $d \equiv 7(\bmod 8), d \equiv 5(\bmod 6)$ and $d \not \equiv 7(\bmod 14)$. Assume Conjectures 2.1 and 2.2. Then there exists a constant depending on $K$, namely $B_{K}$, such that for all $p>B_{K}$ the Fermat equation $x^{p}+y^{p}+z^{p}=0$ doesn't have any nontrivial solutions. We refer to this as "the asymptotic Fermat's Last Theorem holds for K."

Density Theorem 3. Assuming Conjectures 2.1 and 2.2 the asymptotic Fermat's Last Theorem holds for $5 / 6$ of the imaginary quadratic number fields.

As mentioned in the Introduction our results will be a generalization of the main theorem in [8. Therefore we follow their expostion and proofs very closely. We try to avoid repetition however when necessary we include the statements and/or proofs of the results proved in [8].

\section{Acknowledgements}

We thank Professor Samir Siksek for useful discussions and helpful comments.

Both authors are supported by TÜBİTAK (Turkish National and Scientific Research Council) Research Grant 117F045. 


\section{Preliminaries}

In this section we discuss modular forms and set up the terminology and notation which are necessary to state the conjectures assumed in the main theorem. For more details we refer to Sections 2 and 3 of [8] and the references therein.

\subsection{Background about Eigenforms and Galois Representations}

Let $K$ be a number field with ring of integers $\mathcal{O}_{K}$ and $\mathfrak{N}$ be an ideal of $\mathcal{O}_{K}$. A weight two complex eigenform $f$ over $K$ of degree $i$ and level $\mathfrak{N}$ is a ring homomorphism $f: \mathbb{T}_{\mathbb{C}}^{(i)}(\mathfrak{N}) \rightarrow \mathbb{C}$ where $T_{\mathbb{C}}^{(i)}(\mathfrak{N})$ is the commutative $\mathbb{Z}$-algebra inside the endomorphism algebra of $H^{i}\left(Y_{0}(\mathfrak{N}), \mathbb{C}\right)$ generated by the Hecke operators $T_{\mathfrak{q}}$ for every prime $\mathfrak{q}$ not dividing $\mathfrak{N}$. For the precise definition of the locally symmetric space $Y_{0}(\mathfrak{N})$ we refer to [8]. Similarly for primes $p$ that are unramified in $K$ and relatively prime to $\mathfrak{N}$ one can define the weight two mod $p$ eigenform $\theta$ over $K$ of degree $i$ and level $\mathfrak{N}$ as a ring homomorphism $\theta: \mathbb{T}_{\overline{\mathbb{F}}_{p}}^{(i)}(\mathfrak{N}) \rightarrow \overline{\mathbb{F}}_{p}$. In this case only the Hecke operators $T_{\mathfrak{q}}$ are considered for the primes $\mathfrak{q}$ relatively prime to $p \mathfrak{N}$.

Let $\theta$ be a mod $p$ eigenform of level $\mathfrak{N}$ and degree $i$. Let $\mathbb{Q}_{\mathfrak{f}}$ be the number field generated by the values of $\mathfrak{f}$. If there is a complex eigenform $\mathfrak{f}$ of degree $i$ and level $\mathfrak{N}$ and a prime ideal $\mathfrak{p}$ of $\mathbb{Q}_{\mathfrak{f}}$ lying over $p$ such that $\theta\left(T_{\mathfrak{q}}\right) \equiv \mathfrak{f}\left(\bar{T}_{\mathfrak{q}}\right) \bmod \mathfrak{p}$ for every prime $\mathfrak{q}$ of $K$ coprime to $p \mathfrak{N}$, we say that $\theta$ lifts to a complex eigenform.

The following result is proved by Şengün and Siksek in [8].

Proposition. (Proposition 2.1, [8]) There is an integer B, depending only on $\mathfrak{N}$, such that for any prime $p>B$, every $\bmod p$ eigenform of level $\mathfrak{N}$ lifts to a complex one.

\subsection{Conjectures}

One of the conjectures assumed in the main theorem is a special case of Serre's modularity conjecture over number fields, stated below:

Conjecture 2.1. (Conjecture 3.1 in [8]) Let $\rho: G_{K} \rightarrow G L_{2}\left(\overline{\mathbb{F}}_{p}\right)$ be an odd, irreducible, continuous representation with Serre conductor $\mathfrak{N}$ and trivial character. Assume that $p$ is unramified in $K$ and that $\left.\bar{\rho}\right|_{G_{K_{\mathfrak{p}}}}$ arises from a finite-flat group scheme over $\mathcal{O}_{K_{\mathfrak{p}}}$ for every prime $\mathfrak{p} \mid p$. Then there is a weight $2 \bmod p$ eigenform $\theta$ over $K$ of level $\mathfrak{N}$ such that for all primes $\mathfrak{q}$ coprime to $p \mathfrak{N}$, we have

$$
\operatorname{Tr}\left(\bar{\rho}\left(\operatorname{Frob}_{\mathfrak{q}}\right)\right)=\theta\left(T_{\mathfrak{q}}\right) .
$$

Additionally, we will make use of a special case of a fundamental conjecture from Langlands Programme.

Conjecture 2.2. (Conjecture 4.1 in [8]) Let $\mathfrak{f}$ be a weight 2 complex eigenform over $K$ of level $\mathfrak{N}$ that is non-trivial and new. If $K$ has some real place, then there exists an elliptic curve $E_{\mathfrak{f}} / K$ of conductor $\mathfrak{N}$ such that

$$
\# E_{\mathfrak{f}}\left(\mathcal{O}_{K} / \mathfrak{q}\right)=1+\operatorname{Norm}(\mathfrak{q})-\mathfrak{f}\left(T_{\mathfrak{q}}\right) \text { for all } \mathfrak{q} \nmid \mathfrak{N} .
$$

If $K$ is totally complex, then there exists either an elliptic curve $E_{\mathfrak{f}}$ of conductor $\mathfrak{N}$ satisfying (2.1) or a fake elliptic curve $A_{\mathfrak{f}} / K$, of conductor $\mathfrak{N}^{2}$, such that

$$
\# A_{\mathfrak{f}}\left(\mathcal{O}_{K} / \mathfrak{q}\right)=\left(1+\operatorname{Norm}(\mathfrak{q})-\mathfrak{f}\left(T_{\mathfrak{q}}\right)\right)^{2} \quad \text { for all } \quad \mathfrak{q} \nmid \mathfrak{N} .
$$




\section{Frey Curve and Related Facts}

In this section we collect some facts related to the Frey curve associated to a proposed solution of the Fermat equation 1.1 and the associated Galois representation.

Let $G_{K}$ be the absolute Galois group of $K$ and $E$ be an elliptic curve over a number field $K$. Then

$$
\bar{\rho}_{E, p}: G_{K} \longrightarrow \operatorname{Aut}(E[p]) \cong \mathrm{GL}_{2}\left(\mathbb{F}_{p}\right)
$$

denotes the $\bmod p$ Galois representation of $E$.

The below lemma can be found in [8]. We include its statement here for the convenience of the reader.

Lemma 3.1. Let $E$ be an elliptic curve over $K$ with $j$-invariant $j$. Let $p \geq 5$ be a rational prime. Let $\mathfrak{q} \nmid p$ be a prime of $K$.

(i) If $v_{\mathfrak{q}}(j) \geq 0$ (i.e. E has potentially good reduction at $\mathfrak{q}$ ) then the size of $\bar{\rho}_{E, p}\left(I_{\mathfrak{q}}\right)$ divides 24 .

(ii) Suppose $v_{\mathfrak{q}}(j)<0$ (i.e. E has potentially multiplicative reduction at $\mathfrak{q}$ ).

- If $p \nmid v_{\mathfrak{q}}(j)$ then the size of $\bar{\rho}_{E, p}\left(I_{\mathfrak{q}}\right)$ is either $p$ or $2 p$.

- If $p \mid v_{\mathfrak{q}}(j)$ then the size of $\bar{\rho}_{E, p}\left(I_{\mathfrak{q}}\right)$ is 1 or 2 .

Let $T$ and $U$ be the sets defined in the introduction i.e. let $\mathcal{S}_{K}$ be the set of prime ideals of $\mathcal{O}_{K}$ and $R=\operatorname{Rad}(A B C)$. Then $S=\left\{\mathfrak{P} \in \mathcal{S}_{K}: \mathfrak{P} \mid 2 R\right\}, T=\left\{\mathfrak{P} \in \mathcal{S}_{K}: \mathfrak{P} \mid 2\right\}$ and $U=\{\mathfrak{P} \in$ $T: f(\mathfrak{P} / 2)=1\}$. Further, suppose that $U \neq \emptyset$. Let $p$ be an odd prime and $(a, b, c) \in K^{3}$ be a non-trivial solution to the Fermat equation 1.1. Write

$$
\mathcal{G}_{a, b, c}=a \mathcal{O}_{K}+b \mathcal{O}_{K}+c \mathcal{O}_{K}
$$

which we can think of as the greatest common divisor of $a, b, c$. We denote the class of $\mathcal{G}_{a, b, c}$ in the class group of $K$, by $[a, b, c]$. Let $\mathfrak{c}_{1}, \ldots, \mathfrak{c}_{h}$ denote the ideal classes of $K$. Since every ideal class contains infinitely many prime ideals, for each class $\mathfrak{c}_{i}$ we can fix a prime ideal $\mathfrak{m}_{i}$ coprime to $2 R$ with the smallest possible norm reperesenting $\mathfrak{c}_{i}$. The set $\mathcal{H}=\left\{\mathfrak{m}_{i}, \ldots, \mathfrak{m}_{h}\right\}$ denotes our fixed choice of odd prime ideals representing the class group. Note that it is possible to scale $(a, b, c)$ so that it remains integral and $\mathcal{G}_{a, b, c}=\mathfrak{m} \in \mathcal{H}$. For a solution $(a, b, c)$ of the Fermat equation 1.1, we associate the Frey elliptic curve

$$
E=E_{a, b, c}: Y^{2}=X\left(X-A a^{p}\right)\left(X+B b^{p}\right) .
$$

Lemma 3.2. Let $\mathfrak{P} \in U$ and suppose $p>4 v_{\mathfrak{P}}(2)$. Then

(i) E has potentially multiplicative reduction at $\mathfrak{P}$;

(ii) $p$ divides the size of $\bar{\rho}_{E, p}\left(I_{\mathfrak{P}}\right)$ where $I_{\mathfrak{P}}$ denotes the inertia subgroup $G_{K}$ at $\mathfrak{P}$.

Proof. Since $\mathcal{G}_{a, b, c}=\mathfrak{m}$ is relatively prime to $2 R$ and is in $\mathcal{H}$, which consists of odd prime ideals, we have $\mathfrak{m} \nmid 2$ and $\mathfrak{m} \nmid R$. Furthermore, $\mathfrak{P} \nmid R$ implies that $\mathfrak{P}$ divides at most one of $a, b, c$. Say $\mathfrak{P} \nmid a b c$, then we get, $0=A a^{p}+B b^{p}+C c^{p} \equiv 1+1+1 \bmod \mathfrak{P}$. Since the residue field of any element of $U$ is $\mathbb{F}_{2}$, this is a contradiction. Therefore, we see that $\mathfrak{P}$ divides precisely one of $a, b, c$. Now, we permute $a, b, c$ so that $\mathfrak{P} \mid b$. The Frey curve associated to $(b, a, c)$ is

$$
E^{\prime}=E_{b, a, c}: Y^{2}=X\left(X-A b^{p}\right)\left(X+B a^{p}\right) .
$$


For $E$ and $E^{\prime}$ we will compute the corresponding $j$ and $j^{\prime}$. The expressions for $j$ and $j^{\prime}$ in terms of $A, B, C$ and $a, b, c$ are

$$
j=j^{\prime}=2^{8} \cdot \frac{\left(C^{2} c^{2 p}-A B a^{p} b^{p}\right)^{3}}{A^{2} B^{2} C^{2} a^{2 p} b^{2 p} c^{2 p}}
$$

Since $\mathfrak{P} \nmid 2 R$, we know that $\mathfrak{P} \nmid A B C$. Therefore, it follows that $v_{\mathfrak{P}}(j)=8 v_{\mathfrak{P}}(2)-2 p v_{\mathfrak{P}}(b)$ and $v_{\mathfrak{P}}(j)=8 v_{\mathfrak{P}}(2)-2 p v_{\mathfrak{P}}(a)$. By assumption $p>4 v_{\mathfrak{P}}(2)$ which implies that $v_{\mathfrak{P}}(j)<0$. Hence, $E$ has multiplicative reduction at $\mathfrak{P}$. Furthermore, $p \nmid v_{\mathfrak{P}}(j)$. The rest of the lemma follows from the part (ii) of Lemma 3.1 .

Lemma 3.3. Suppose $p \geq 5$ and $\mathfrak{m} \nmid p$. Then the size of $\bar{\rho}_{E, p}\left(I_{\mathfrak{m}}\right)$ divides 24 .

Proof. We know that $v_{\mathfrak{m}}(a), v_{\mathfrak{m}}(b)$ and $v_{\mathfrak{m}}(c)$ are all positive as $\mathcal{G}_{a, b, c}=\mathfrak{m}$. Furthermore, since $A a^{p}+B b^{p}+C c^{p}=0$, we have $v_{\mathfrak{m}}\left(-C c^{p}\right)=v_{\mathfrak{m}}\left(A a^{p}+B b^{p}\right) \geq \min \left\{v_{\mathfrak{m}}\left(A a^{p}\right), v_{\mathfrak{m}}\left(B b^{p}\right)\right\}$ with equality if $v_{\mathfrak{m}}\left(A a^{p}\right) \neq v_{\mathfrak{m}}\left(B b^{p}\right)$, which implies that at least two of $v_{\mathfrak{m}}\left(A a^{p}\right), v_{\mathfrak{m}}\left(B b^{p}\right), v_{\mathfrak{m}}\left(C c^{p}\right)$ are equal. Then, either we have

$$
v_{\mathfrak{m}}\left(A a^{p}\right)=v_{\mathfrak{m}}\left(-C c^{p}\right)=k, \quad v_{\mathfrak{m}}\left(B b^{p}\right)=k+t
$$

or

$$
v_{\mathfrak{m}}\left(B b^{p}\right)=v_{\mathfrak{m}}\left(-C c^{p}\right)=k, \quad v_{\mathfrak{m}}\left(A a^{p}\right)=k+t
$$

where $k \geq 1$ and $t \geq 0$. From the expression of $j$, we get

$$
\begin{aligned}
v_{\mathfrak{m}}(j) \geq & 3 \min \left\{2 v_{\mathfrak{m}}\left(C c^{p}\right), v_{\mathfrak{m}}\left(A a^{p}\right)+v_{\mathfrak{m}}\left(B b^{p}\right)\right\} \\
& -2\left(v_{\mathfrak{m}}\left(A a^{p}\right)+v_{\mathfrak{m}}\left(B b^{p}\right)+v_{\mathfrak{m}}\left(C c^{p}\right)\right) .
\end{aligned}
$$

If $t=0$, then $v_{\mathfrak{m}}(j)=0$ and thus the lemma follows from the part ( $\left.i\right)$ of Lemma 3.1, Now, suppose that $t \geq 1$. In this case, we get $v_{\mathfrak{m}}(j)=-2 t$. If we can show that $p \mid-2 t$, by Lemma 3.1, the size of $\bar{\rho}_{E, p}\left(I_{\mathfrak{m}}\right)$ is 1 or 2 , completing the proof.

Since $k=v_{\mathfrak{m}}\left(A a^{p}\right)=v_{\mathfrak{m}}(A)+p v_{\mathfrak{m}}(a)$ and $k+t=v_{\mathfrak{m}}\left(B b^{p}\right)=v_{\mathfrak{m}}(B)+p v_{\mathfrak{m}}(b)$, we have $t=$ $v_{\mathfrak{m}}(B)-v_{\mathfrak{m}}(A)+p\left(v_{\mathfrak{m}}(b)-v_{\mathfrak{m}}(a)\right)$. We also know that $\mathfrak{m} \nmid R$, so $\mathfrak{m} \nmid A B C$. Hence, $\mathfrak{m}$ divides none of the constants $A, B, C$ which gives $v_{\mathfrak{m}}(A)=v_{\mathfrak{m}}(B)=v_{\mathfrak{m}}(C)=0$.

Let $E$ be an elliptic curve over a number field having semistable reduction away from a set of primes $\mathcal{P}$. If $p \geq 5$ is not divisible by any prime in $\mathcal{P}$ then by the theory of Weil pairing on $E[p]$ the determinant of the mod $p$ Galois representation associated to $E$, namely $\bar{\rho}_{E, p}$ has determinant equal to the cyclotomic character, [see [1, p.21]. Therefore the representation $\bar{\rho}_{E, p}$ is odd. Recall that a representation $\bar{\rho}: G_{K} \rightarrow \mathrm{GL}_{2}\left(\overline{\mathbb{F}}_{p}\right)$ is odd if the determinant of every complex conjugation is -1 . Given a number field $K$, we obtain a complex conjugation for every real embedding $\sigma: K \hookrightarrow \mathbb{R}$ and every extension $\tilde{\sigma}: \bar{K} \hookrightarrow \mathbb{C}$ of $\sigma$ as $\tilde{\sigma}^{-1} \iota \tilde{\sigma} \in G_{K}$ where $\iota$ is the usual complex conjugation. If the number field $K$ has no real embeddings to begin with, then we immediately say that $\bar{\rho}$ is odd.

The following results gives some of the reduction properties of the Frey elliptic curve.

Lemma 3.4. The Frey curve $E$ associated to the Fermat equation 1.1 is semistable away from $S \cup\{\mathfrak{m}\}$, where $\mathfrak{m}=\mathcal{G}_{a, b, c}$.

Proof. Let $\mathfrak{q} \notin S \cup\{\mathfrak{m}\}$ be a prime of $K$. The invariants $c_{4}$ and $\Delta$ of the model $E$ given in (3.1) are

$$
c_{4}=2^{4}\left(\left(B b^{p}\right)^{2}-A a^{p} C c^{p}\right), \quad \Delta=2^{4}(A B C)^{2}(a b c)^{2 p} .
$$

Suppose that $\mathfrak{q}$ divides $\Delta$. None of $2, A, B$ and $C$ is divisible by $\mathfrak{q}$ since $\mathfrak{q} \nmid 2 R$ which implies that $a b c$ is divisible by $\mathfrak{q}$. On the other hand, $\mathfrak{q}$ can divide at most one of $a, b, c$. If two of them were 
divisible by $\mathfrak{q}$, then $A a^{p}+B b^{p}+C c^{p}=0$ would lead to $\mathfrak{q}=\mathfrak{m}$ which is a contradiction. Therefore, $\left(\left(B b^{p}\right)^{2}-A a^{p} C c^{p}\right)$ is not divisible by $\mathfrak{q}$ and so $\mathfrak{q} \nmid c_{4}$. This also gives $v_{\mathfrak{q}}\left(c_{4}\right)=0$. Hence, the given model is minimal and $E$ is semistable at $\mathfrak{q}$.

Corollary 3.5. Let $E$ be the Frey curve associated to the Fermat equation 1.1, defined over the number field $K$. Let $p \geq 5$ and $p$ is not divisible by any $\mathfrak{q} \in S \cup\{\mathfrak{m}\}$ and let $\bar{\rho}_{E, p}$ denote the $\bmod p$ Galois representation associated to $E$. Then:

1. The determinant of $\bar{\rho}_{E, p}$ is the mod $p$ cyclotomic character, hence it is odd.

2. Serre conductor of $\bar{\rho}_{E, p}, \mathfrak{N}$, is supported on $S \cup\{\mathfrak{m}\}$ and belongs to a finite set that depends only on the field $K$.

3. $\bar{\rho}_{E, p}$ is finite flat at every $\mathfrak{q}$ over $p$.

Proof. Part (1) follows from the paragraph preceding Lemma 3.4

For Parts (2) and (3) we use $\Delta=2^{4}(A B C)^{2}(a b c)^{2 p}$ computed in Lemma 3.4. Now, $v_{\mathfrak{q}}(\Delta)=$ $4 v_{\mathfrak{q}}(2)+2\left(v_{\mathfrak{q}}(A)+v_{\mathfrak{q}}(B)+v_{\mathfrak{q}}(C)\right)+2 p\left(v_{\mathfrak{q}}(a)+v_{\mathfrak{q}}(b)+v_{\mathfrak{q}}(c)\right)=2 p v$. where $v$ is exactly one of the $v_{\mathfrak{q}}(a), v_{\mathfrak{q}}(b), v_{\mathfrak{q}}(c)$. Hence, $p \mid v_{\mathfrak{q}}(\Delta)$. We deduce that $\bar{\rho}$ is unramified at $\mathfrak{q}$ if $\mathfrak{q} \nmid p$ and finite flat at $\mathfrak{q}$ if $\mathfrak{q} \mid p$ (c.f.[6]). Finally, we show that there can be only finitely many Serre conductors $\mathfrak{N}$. Only the primes in $\mathfrak{q} \in S \cup\{\mathfrak{m}\}$ can divide $\mathfrak{N}$. As $\mathfrak{N}$ divides the conductor $N$ of $E, v_{\mathfrak{q}}(\mathfrak{N}) \leq v_{\mathfrak{q}}(N) \leq 2+3 v_{\mathfrak{q}}(3)+6 v_{\mathfrak{q}}(2)$ by [Silverman,theorem IV.10.4]. Hence, there can be only finitely many Serre conductors and they only depend on $K$ since $\mathfrak{m} \in \mathcal{H}$ and $\mathcal{H}$ only depends on $K$.

\section{Level Lowering}

In this section we will be relating the Galois representation attached to Frey curve with another representation of lower level. The following result is the main theorem of the section:

Theorem 4.1. Let $K$ be a number field. Assume Conjecture 2.1] and Conjecture [2.2. Suppose that $U \neq \emptyset$. Then there is a constant $B_{K}$ depending only on $K$ such that the following holds. Let $(a, b, c) \in \mathcal{O}_{K}^{3}$ be a non-trivial solution to the Fermat equation with exponent $p>B_{K}$, and suppose that it is scaled so that $\mathcal{G}_{a, b, c}=\mathfrak{m} \in \mathcal{H}$. Let $E / K$ be the associated Frey curve defined in (3.1). Then there is an elliptic curve $E^{\prime} / K$ such that the following statements hold:

(i) $E^{\prime}$ has good reduction away from $S \cup\{\mathfrak{m}\}$, and potentially good reduction away from $S$.

(ii) $E^{\prime}$ has full 2-torsion.

(iii) $\bar{\rho}_{E, p} \sim \bar{\rho}_{E^{\prime}, P}$.

(iv) For $\mathfrak{P} \in U$ we have $v_{\mathfrak{P}}\left(j^{\prime}\right)<0$ where $j^{\prime}$ is the $j$-invariant of $E^{\prime}$.

We will give the proof of this Theorem in Section 4.1 after stating the necessary lemmas.

\subsection{Surjectivity of Galois representations}

The following is Proposition 6.1 from Şengün and Siksek [8]. We include its statement for the convenience of the reader but we will omit its proof and refer to [8] instead. 
Proposition 4.2. Let $L$ be a Galois number field and let $\mathfrak{q}$ be a prime of $L$. There is a constant $B_{L, \mathfrak{q}}$ such that the following is true. Let $p>B_{L, \mathfrak{q}}$ be a rational prime. Let $E / L$ be an elliptic curve that is semistable at all $\mathfrak{p} \mid p$ and having potentially multiplicative reduction at $\mathfrak{q}$. Then $\bar{\rho}_{E, p}$ is irreducible.

By applying the above proposition to the Frey curve, we get the following corollary.

Corollary 4.3. Let $K$ be a number field, and suppose that $U \neq \emptyset$. There is a constant $C_{K}$ such that if $p>C_{K}$ and $(a, b, c) \in \mathcal{O}_{K}^{3}$ is a non-trivial solution to the Fermat equation with exponent $p$, and scaled so that $\mathcal{G}_{a, b, c} \in \mathcal{H}$, then $\bar{\rho}_{E, p}$ is surjective, where $E$ is the Frey curve given in (3.1).

Proof. Suppose $\mathfrak{P} \in U$, then $E$ has potentially multiplicative reduction at $\mathfrak{P}$ by Lemma 4.2. Also, $E$ is semistable away from the primes in $S \cup \mathcal{H}$ from Lemma 4.4. Let $L$ be the Galois closure of $K$, and let $\mathfrak{q}$ be a prime of $L$ above $\mathfrak{P}$. Now, by applying Proposition 5.1 , we get a constant $B_{L, \mathfrak{q}}$ such that $\bar{\rho}_{E, p}\left(G_{L}\right)$ is irreducible whenever $p>B_{L, \mathfrak{q}}$. Now, we observe that $B_{L, \mathfrak{q}}$ depends on only $K$ since $\mathfrak{p}$ is a prime of $L$ above $\mathfrak{P}$ which is a prime of $K$ above 2 . We denote $B_{L, \mathfrak{q}}$ by $C_{K}$. If necessary, enlarge $C_{K}$ so that $C_{K}>4 v_{\mathfrak{p}}(2)$. Now, we apply part (ii) of Lemma 4.2 and see that the image of $\bar{\rho}_{E, p}$ contains an element of order $p$. It is known that any subgroup of $\mathrm{GL}_{2}\left(\mathbb{F}_{p}\right)$ having an element of order $p$ is either reducible or contains $\mathrm{SL}_{2}\left(\mathbb{F}_{p}\right)$. As $p>C_{K}>4 v_{\mathfrak{P}}(2)$, the image contains $\mathrm{SL}_{2}\left(\mathbb{F}_{p}\right)$. Finally, we can ssume that $K \cap \mathbb{Q}\left(\zeta_{p}\right)=\mathbb{Q}$ by taking $C_{K}$ large enough if needed. Hence, $\chi_{p}=\operatorname{det}(\bar{\rho})_{E, p}$ is surjective.

\subsection{Proof of Theorem 4.1}

In this section, Theorem 4.1 will be proven. We continue with the notations introduced in the statement of Theorem 4.1 and the assumptions of the theorem.

Lemma 4.4. There is a non-trivial, new (weight 2) complex eigenform $\mathfrak{f}$ which has an associated elliptic curve $E_{\mathfrak{f}} / K$ of conductor $\mathfrak{N}^{\prime}$ dividing $\mathfrak{N}$.

Proof. To start with, we show the existence of such an eigenform $\mathfrak{f}$ of level $\mathfrak{N}$ supported only on $S \cup\{\mathfrak{m}\}$.

By Corollary 4.3, the representation $\bar{\rho}_{E, p}: G_{K} \rightarrow G L_{2}\left(\mathbb{F}_{p}\right)$ is surjective hence is absolutely irreducible for $p>C_{K}$. Now, we apply Conjecture 2.1 to deduce that there is a weight $2 \bmod p$ eigenform $\theta$ over $K$ of level $\mathfrak{N}$ such that for all primes $\mathfrak{q}$ coprime to $p \mathfrak{N}$, we have

$$
\operatorname{Tr}\left(\bar{\rho}_{E, p}\left(\operatorname{Frob}_{\mathfrak{q}}\right)\right)=\theta\left(T_{\mathfrak{q}}\right) .
$$

We know from Corollary 3.5 that there are only finitely many possible levels $\mathfrak{N}$. Thus, by taking $p$ large enough, there is a weight 2 complex eigenform $\mathfrak{f}$ with level $\mathfrak{N}$ which is a lift of $\theta$ for any level $\mathfrak{N}$. Note that since there are only finitely many such eigenforms $\mathfrak{f}$ and they depend only on $K$, from now on we can suppose that every constant depending on these eigenforms depends only on $K$.

Next, we recall that if $\mathbb{Q}_{\mathfrak{f}}=\mathbb{Q}$ then there is a constant $C_{\mathfrak{f}}$ depending only on $\mathfrak{f}$ such that $p<C_{\mathfrak{f}}$ ([8], Lemma 7.2). Therefore, by taking $p$ sufficiently large, we assume that $\mathbb{Q}_{\mathfrak{f}}=\mathbb{Q}$. In order to apply Conjecture 2.2. we need to show that $\mathfrak{f}$ is non-trivial and new. As $\bar{\rho}_{E, p}$ is irreducible, the eigenform $\mathfrak{f}$ is non-trivial. If $\mathfrak{f}$ is new, we are done. If not, we can replace it with an equivalent new eigenform of smaller level. Therefore, we can take $\mathfrak{f}$ new with level $\mathfrak{N}^{\prime}$ dividing $\mathfrak{N}$. Finally, we apply Conjecture 2.2 and obtain that $\mathfrak{f}$ either has an associated elliptic curve $E_{\mathfrak{f}} / K$ of conductor $\mathfrak{N}^{\prime}$, or has an associated fake elliptic curve $A_{\mathfrak{f}} / K$ of conductor $\mathfrak{N}^{2}$. 
By Lemma 4.5, if $p>24$, then $\mathfrak{f}$ has an associated elliptic curve $E_{\mathfrak{f}}$.

As a result, we can assume that $\bar{\rho}_{E, p} \sim \bar{\rho}_{E^{\prime}, p}$ where $E^{\prime}=E_{\mathfrak{f}}$ is an elliptic curve with conductor $\mathfrak{N}^{\prime}$ dividing $\mathfrak{N}$. This completes the proof of the claim.

Lemma 4.5. If $p>24$, then $\mathfrak{f}$ has an associated elliptic curve $E_{\mathfrak{f}}$.

Proof. Let $\mathfrak{P} \in U$ as we assume $U \neq \emptyset$. By Lemma 4.2, $p \mid \# \bar{\rho}_{E, p}\left(I_{\mathfrak{P}}\right)$ whenever $p>4 v_{\mathfrak{P}}(2)$. Assume for a contradiction that $\mathfrak{f}$ corresponds to a fake elliptic curve $A_{\mathfrak{f}}$, then $\# \bar{\rho}_{A_{\mathfrak{f}}, p}\left(I_{\mathfrak{P}}\right) \leq 24$ by Theorem 4.2 of [8]. (Note that here, $\bar{\rho}_{A_{\mathfrak{f}}, p}$ is the 2-dimensional representation defined in Section 4 , Equation 3 of [8]). Thus $\# \bar{\rho}_{E, p}\left(I_{\mathfrak{P}}\right) \leq 24$ due to $\bar{\rho}_{A_{\mathfrak{f}}, p} \sim \bar{\rho}_{E, p}$ leading to a contradiction when $p>24$.

We can now give the proof of Theorem 4.1 ,

Proof of Theorem 4.1: Lemma 4.5 gives us that if $p>24$, then $\mathfrak{f}$ has an associated elliptic curve $E_{\mathfrak{f}}$. Therefore, by Lemma 4.4 we can assume that $\bar{\rho}_{E, p} \sim \bar{\rho}_{E^{\prime}, p}$ where $E^{\prime}=E_{\mathfrak{f}}$ is an elliptic curve of conductor $\mathfrak{N}^{\prime}$ dividing $\mathfrak{N}$.

The elliptic curve $E^{\prime}$ does not need to have full 2-torsion. We will refer to [8] to overcome this problem. If $E^{\prime}$ does not have full 2-torsion, we can always find another elliptic curve $E^{\prime \prime}$ with full 2-torsion that is 2-isogenous to $E^{\prime}$ for big enough prime $p$, see Lemma 7.4, Lemma 7.5 in 8 .

Now, suppose $E^{\prime}$ is 2-isogenous to an elliptic curve $E^{\prime \prime}$. As the isogeny induces an isomorphism $E^{\prime}[p] \cong E^{\prime \prime}[p]$ of Galois modules $(p \neq 2), \bar{\rho}_{E, p} \sim \bar{\rho}_{E^{\prime}, p} \sim \bar{\rho}_{E^{\prime \prime}, p}$ completing the proof of (iii). After possibly replacing $E^{\prime}$ by $E^{\prime \prime}$, we can suppose that $E^{\prime}$ has full 2-torsion giving us (ii).

It remains to prove (i) and (iv). Let $\mathfrak{P} \in U$, then $p$ divides the size of $\bar{\rho}_{E, p}\left(I_{\mathfrak{P}}\right)$ by Lemma 3.2 , Now, Lemma 3.1 implies that $v_{\mathfrak{P}}\left(j^{\prime}\right)<0$ for $\mathfrak{P} \in U$ since the sizes of $\bar{\rho}_{E, p}\left(I_{\mathfrak{P}}\right)$ and $\bar{\rho}_{E^{\prime}, p}\left(I_{\mathfrak{P}}\right)$ are equal. This proves (iv). Finally, we need to show that $E^{\prime}$ has potentially good reduction at $\mathfrak{m}$. Assume the contrary that $E^{\prime}$ has a multiplicative reduction at $\mathfrak{m}$. Then, by Lemma 3.1, we have $p \mid \# \bar{\rho}_{E^{\prime}, p}\left(I_{\mathfrak{m}}\right)$ for every $p>\left|v_{\mathfrak{m}}\left(j^{\prime}\right)\right|$. On the other hand, the size of $\bar{\rho}_{E^{\prime}, p}\left(I_{\mathfrak{m}}\right)$ is less than 24 by Lemma 3.3 giving a contradiction for large $p$.

\section{Relation with S-unit equation and elliptic curves}

So far, we have proved that assuming a non-trivial solution to the generalized Fermat equation yields an elliptic curve $E^{\prime}$ with full 2-torsion having potentially good reduction away from the set $S$. In order to prove our Main Theorem, we relate such elliptic curves $E^{\prime}$ to the solutions of the $S$-unit equation 1.2 via $\lambda$-invariants of elliptic curves (see, e.g., [7, pp. 53-55). For more details, we refer the reader to the Section 5 of [3].

Let $E^{\prime}$ bea an elliptic curve over $K$ with full 2-torsion which has a model

$$
E^{\varsigma}: Y^{2}=\left(X-e_{1}\right)\left(X-e_{2}\right)\left(X-e_{3}\right),
$$

where $e_{1}, e_{2}, e_{3}$ are all distinct. Then their cross ratio $\lambda=\frac{e_{3}-e_{1}}{e_{2}-e_{1}}$ is an element of $\mathbb{P}^{1}(K)-\{0,1, \infty\}$. Conversely we can obtain any $\lambda$ in $\mathbb{P}^{1}(K)-\{0,1, \infty\}$ as a cross ratio of three distinct elements of $K$. Now, let us denote the symmetric group on three letters by $\mathfrak{S}_{3}$. The action of $\mathfrak{S}_{3}$ on the set $\left\{e_{1}, e_{2}, e_{3}\right\}$ can be extented to a well-defined action on $\mathbb{P}^{1}(K)-\{0,1, \infty\}$ via the cross ratio. Hence, we can view $\mathfrak{S}_{3}$ as the below subgroup of $\operatorname{PGL}_{2}(K)$ :

$$
\mathfrak{S}_{3}=\{z, 1 / z, 1-z, 1 /(1-z), z / z-1,(z-1) / z\} .
$$


Under the action of $\mathfrak{S}_{3}$, the orbit of $\lambda$ in $\mathbb{P}^{1}(K)-\{0,1, \infty\}$, called $\lambda$-invariants corresponds to the following set:

$$
\left\{\lambda, \frac{1}{\lambda}, 1-\lambda, \frac{1}{1-\lambda}, \frac{\lambda}{\lambda-1}, \frac{\lambda-1}{\lambda}\right\} .
$$

Every elliptic curve $E^{\prime}$ of the form (5.1) is isomorphic (over $\bar{K}$ ) to an elliptic curve in the Legendre form $E_{\lambda}: Y^{2}=X(X-1)(X-\lambda)$ for $\lambda \in \mathbb{P}^{1}(K)-\{0,1, \infty\}$ whose $j$-invariant is

$$
j(\lambda)=2^{8} \frac{\left(\lambda^{2}-\lambda+1\right)^{3}}{\lambda^{2}(1-\lambda)^{2}} .
$$

Moreover, there is a one-to-one correspondence between the $\bar{K}$-isomorphism classes of the Legendre elliptic curves and the $\mathfrak{S}_{3}$-orbits of elements of $\lambda \in \mathbb{P}^{1}(K)-\{0,1, \infty\}$.

The following result is Lemma 7.1 of [3], we state it here for the convenience of the reader.

Lemma 5.1. Suppose the size of $S$ is 2 . Let $(\lambda, \mu) \in \Lambda_{S}$, where $\Lambda_{S}$ is the set of solutions to the $S$-unit equation 1.2. Then there is some element $\sigma \in \mathfrak{S}_{3}$ such that $(\lambda, \mu)^{\sigma}=\left(\lambda^{\prime}, \mu^{\prime}\right)$ satisfies $\lambda^{\prime}, \mu^{\prime} \in \mathcal{O}_{K}$.

\section{Proof of the Main Theorem}

In this section, we will prove our main theorem. Our proof closely follows the proof of Theorem 3 in [4]. Let $K$ be a number field satisfying Conjectures 2.1 and 2.2 and $S, U, V$ be the sets in (11) with $U \neq \emptyset$. Let $B_{K}$ be as in Theorem 4.1, and let $(a, b, c)$ be a non-trivial solution to the Fermat equation 1.1, which is scaled so that $\mathcal{G}_{a, b, c}=\mathfrak{m}$ where $\mathfrak{m} \in \mathcal{H}$, with exponent $B_{K}$. We now apply Theorem 4.1 and obtain an elliptic curve $E^{\prime} / K$ having full 2-torsion and potentially good reduction away from $S$ with $j$-invariant $j^{\prime}$ satisfying $v_{\mathfrak{P}}\left(j^{\prime}\right)<0$ for all $\mathfrak{P} \in U$.

Let $\lambda$ be any of the $\lambda$-invariants of $E^{\prime}$. As $E^{\prime}$ has potentially good reduction away from $S$, the $j$-invariant $j^{\prime}$ belongs to $\mathcal{O}_{S}$ where $\mathcal{O}_{S}$ is the ring of $S$-integers in $K$. From the equation [5.3, we can deduce that $\lambda \in K$ satisfies a monic polynomial with coefficients in $\mathcal{O}_{S}$ implying $\lambda \in \mathcal{O}_{S}$. On the other hand, notice that $1 / \lambda, \mu:=1-\lambda$ and $1 / \mu$ are solutions of (5.3) and hence elements of $\mathcal{O}_{S}$. Therefore, we conclude that $(\lambda, \mu)$ is a solution of the $S$-unit equation 1.2,

Now, by the assumption of our main theorem, there is some $\mathfrak{P} \in U$ for every such solution $(\lambda, \mu)$ satisfying $\max \left\{\left|v_{\mathfrak{P}}(\lambda)\right|,\left|v_{\mathfrak{P}}(\mu)\right|\right\} \leq 4 v_{\mathfrak{P}}(2)$. Let $t:=\max \left\{\left|v_{\mathfrak{P}}(\lambda)\right|,\left|v_{\mathfrak{P}}(\mu)\right|\right\}$ and further let us express $j^{\prime}$ in terms of $\lambda$ and $\mu$ as:

$$
j^{\prime}=2^{8} \frac{(1-\lambda \mu)^{3}}{(\lambda \mu)^{2}}
$$

Now, if $t=0$, then $v_{\mathfrak{P}}\left(j^{\prime}\right) \geq 8 v_{\mathfrak{P}}(2) \geq 0$ by (6.1). This contradicts with the assumption that $v_{\mathfrak{P}}\left(j^{\prime}\right)<0$ for all $\mathfrak{P} \in U$. Hence, we may suppose $t>0$. The relation $\lambda+\mu=1$ leads to $v_{\mathfrak{P}}(\lambda+\mu) \geq \min \left\{v_{\mathfrak{P}}(\lambda), v_{\mathfrak{P}}(\mu)\right\}$ with equality if $v_{\mathfrak{P}}(\lambda) \neq v_{\mathfrak{P}}(\mu)$. This shows either $v_{\mathfrak{P}}(\lambda)=v_{\mathfrak{P}}(\mu)=$ $-t$, or $v_{\mathfrak{P}}(\lambda)=t$ and $v_{\mathfrak{P}}(\mu)=0$, or $v_{\mathfrak{P}}(\lambda)=0$ and $v_{\mathfrak{P}}(\mu)=t$. Therefore, $v_{\mathfrak{P}}(\lambda \mu)=-2 t<0$ or $v_{\mathfrak{P}}(\lambda \mu)=t>0$. In any of the cases, we have $v_{\mathfrak{P}}\left(j^{\prime}\right) \geq 8 v_{\mathfrak{P}}(2)-2 t \geq 0$, which again yields a contradiction. This completes the proof of the main theorem.

\section{Proof of Density Theorem 1}

To prove the first density result we need to understand the solutions to the $S$-unit equation in the quadratic fields $K$ satisfying the assumptions of the theorem. Using the notation introduced in the 
beginning of the paper we have $S=\{\mathfrak{P}, q\}$ and $T=\{\mathfrak{P}\}$ where $\mathfrak{P}^{2}=2 \mathcal{O}_{K}$. Note that 2 is ramified in $K$ by assumption. First assume that $d>2$. Then the unit group of $\mathcal{O}_{K}$ is $\{ \pm 1\}$. Also the ideal $\mathfrak{P}$ cannot be principal since the equation $a^{2}+d b^{2}=2$ has no solutions in integers.

We claim that if $(\lambda, \mu)$ is a solution to the $S$-unit equation $\lambda+\mu=1$ then $(\lambda, \mu)$ is in the set $\{(-1,2),(1,-2),(1 / 2,1 / 2)\}$. Following [4], these solutions will be called irrelevant solutions, while other solutions will be called relavant solutions. By Lemma 5.1 there is an element $\left(\lambda^{\prime}, \mu^{\prime}\right)$ in the orbit of $(\lambda, \mu)$ such that $\lambda^{\prime}, \mu^{\prime} \in \mathcal{O}_{K}$.

In this section we use Section 7 of [3], most of the results are analogous to results therein. For instance we only give the statement of the following result since its proof is given in [3].

Lemma 7.1. Let $K$ and $S$ be as in the statement of the Density Theorem 1 and let $(\lambda, \mu) \in \Lambda_{S}$. Then $\lambda, \mu \in \mathbb{Q}$ if and only if $(\lambda, \mu)$ belongs to the $\mathfrak{S}_{3}$-orbit $\{(-1,2),(1,-2),(1 / 2,1 / 2)\}$.

This result is following the proof given in [4] and [3] for the imaginary case.

Lemma 7.2. Up to the action of $\mathfrak{S}_{3}$, every relevant $(\lambda, \mu) \in \mathfrak{S}_{3}$ has the form:

$$
\begin{array}{r}
\lambda=\frac{2^{2 r_{1}} q^{2 s_{1}}-q^{2 s_{2}}+1+v \sqrt{-d}}{2}, \mu=\frac{q^{2 s_{2}}-2^{2 r_{1}} q^{2 s_{1}}+1-v \sqrt{-d}}{2}, \\
\text { where } r_{1}, s_{1}, s_{2} \geq 0, s_{1} s_{2}=0, v \in \mathbb{Z}-\{0\} \\
\text { and satisfies }\left(2^{2 r_{1}} q^{2 s_{1}}-q^{2 s_{2}}+1\right)^{2}+d v^{2}=2^{2 r_{1}+2} q^{2 s_{1}} \\
\left(q^{2 s_{2}}-2^{2 r_{1}} q^{2 s_{1}}+1\right)^{2}+d v^{2}=2^{2} q^{2 s_{2}}
\end{array}
$$

Proof. It is clear that if $\lambda, \mu$ satisfy the given equations with the given conditions $(\lambda, \mu)$ is a relevant element of $\Lambda_{S}$.

Conversely assume that $(\lambda, \mu)$ is a relevant element of $\Lambda_{S}$. By Lemmas 5.1 and 7.1 we can assume that $\lambda, \mu \in \mathcal{O}_{K}-\mathbb{Q}$. Since $S=\{2, q\}$ we can write $\lambda=2^{r_{1}} q^{s_{1}} \lambda^{\prime}$ and $\mu=2^{r_{2}} q^{s_{2}} \mu^{\prime}$ where $\lambda^{\prime}, \mu^{\prime}$ are units. Since $\lambda+\mu=1$ we see that $r_{1} r_{2}=s_{1} s_{2}=0$. Without loss of generality we can assume that $r_{2}=0$. Then we get:

$\lambda \bar{\lambda}=2^{2 r_{1}} q^{2 s_{1}}, \mu \bar{\mu}=q^{2 s_{2}}$ where $\bar{x}$ denotes the conjugation for any $x \in K$ and $\lambda+\bar{\lambda}=\lambda \bar{\lambda}-(1-$ $\lambda)(1-\bar{\lambda})+1=\lambda \bar{\lambda}-\mu \bar{\mu}+1=2^{2 r_{1}} q^{2 s_{1}}-q^{2 s_{2}}+1$. Note that $\lambda-\bar{\lambda}=\nu \sqrt{-d}$, where $\nu \in \mathbb{Z}-\{0\}$ since $\lambda \notin \mathbb{Q}$. Combining this with the expression for $\lambda+\bar{\lambda}$ we get the equation given in (7.1). Similarly using the equation $\mu=1-\lambda$ we get the similar expression for $\mu$. The relations 7.3 and 7.4 follow from the identity: $(\lambda+\bar{\lambda})^{2}-(\lambda-\bar{\lambda})^{2}=4 \lambda \bar{\lambda}$ and similar identity for $\mu$.

The following lemma shows that under the given assumptions there are no relevant solutions of the unit equation and finishes the proof of the first density theorem.

Lemma 7.3. Let $d \geq 7$ be a squarefree integer such that $-d \equiv 2,3(\bmod 8), q \geq 29$ be a prime such that $q \equiv 5(\bmod 8)$ and $\left(\frac{d}{q}\right)=-1$. Then there are no relevant elements in $\Lambda_{S}$.

Proof. By Lemma 7.2 we have $s_{1} s_{2}=0$.

- Case 1: Say $s_{1} \geq 0$. Then $s_{2}=0$. Since $q$ doesn't divide $d$, we have by (7.3) $q^{2 s_{1}}$ divides $v^{2}$ i.e. $q^{s_{1}}$ divides $v$. By dividing both sides of (7.3) by $q^{2 s_{1}}$ we get: $2^{4 r_{1}} q^{2 s_{1}}+d\left(\frac{v}{q^{s_{1}}}\right)^{2}=2^{2 r_{1}+2}$. Bringing both sides to modulo $q$ we get: $d\left(\frac{v}{q^{s_{1}}}\right)^{2} \equiv 2^{2 r_{1}+2}(\bmod q)$ i.e. $\left(\frac{d}{q}\right)=1$ which is a contradiction.

Hence $s_{2} \neq 0$ and $s_{1}=0$. 
- Case 2: We know by Case 1 that $s_{1}=0$. Also assume that $s_{2}=0$. If $r_{1} \geq 1$ then $\left(2^{2 r_{1}}\right)^{2} \geq 2^{2 r_{1}+2}$ hence we get a contractiction to equation 7.3 . If $r_{1}=0$ we get $d v^{2}=3$ by (7.3), contradiction again.

- Case 3: We know by Case 1 that $s_{1}=0$ and by Case 2 that $s_{2}>0$. Reducing (7.4) modulo $q^{2 s_{2}}$ we get $\left(2^{2 r_{1}}+1\right)^{2} \equiv-d v^{2}\left(\bmod q^{2 s_{2}}\right)$. Since $\left(\frac{-d}{q}\right)=-1$ we get $q^{s_{2}} \mid v$ and $q^{s_{2}} \mid 2^{2 r_{1}}+1$. Since we assumed that $q \geq 29$ and $q \equiv 5(\bmod 8)$, we get $r_{1} \geq 5$. Write $v=2^{t} w, 2 \nmid w$.

- Case 3a: Say $r_{1}-1 \geq t$. Then $2^{2 r_{1}}-q^{2 s_{2}}+1=2^{t} w^{\prime}$ where $2 \nmid w^{\prime}$. Cancelling out $2^{2 t}$ from both sides of (7.3), we get $w^{\prime 2}+d w^{2} \equiv 0(\bmod 8)$. Since both $w, w^{\prime}$ are odd, their squares have to be 1 modulo 8 . Then we get $-d \equiv 1(\bmod 8)$, contradiction.

- Case 3b: By the previous case we can suppose that $t \geq r_{1}$. By (7.3), $2^{r_{1}}$ divides $\left(2^{r_{1}}-q^{2 s_{2}}+1\right)$ which implies that $2^{r_{1}} \mid\left(1-q^{s_{2}}\right)\left(1+q^{s_{2}}\right)$. Since $q \equiv 5(\bmod 8), q^{s_{2}} \equiv 1$ or $5(\bmod 8)$. Then $2 \mid\left(1+q^{s_{2}}\right)$ and $4 \nmid\left(1+q^{s_{2}}\right)$. Therefore $2^{r_{1}-1} \mid\left(1-q^{s_{2}}\right)$ and since $r_{1}-1 \geq 4$ we get $s_{2}$ is even, say $2 k$. Then similar as above $2^{r_{1}-1} \mid q^{2 k_{2}-1}$ implies that $2^{r_{1}-2} \mid\left(q^{k}-1\right)$ i.e. $q^{k}=M 2^{r_{1}-2}+1$ for some positive integer $M$. We also have seen that $q^{s_{2}} \mid\left(2^{2 r_{1}}+1\right)$ which implies that $q^{s_{2}}=q^{2 k}=M^{2} 2^{2 r_{1}-4}+M 2^{r_{1}-1}+1 \leq 2^{2 r_{1}}+1$ simplifying this inequality we get:

$$
M^{2} 2^{r_{1}-3}+M \leq 2^{r_{1}+1}
$$

this implies that $1 \leq M \leq 3$.

Since $q^{k}=M 2^{r_{1}-2}+1$ and $r_{1} \geq 5, q^{k} \equiv 1(\bmod 8)$. Therefore $k$ must be even, say $2 u$ and we get $\left(q^{u}-1\right)\left(q^{u}+1\right)=M 2^{r_{1}-2}$. The same argument can be applied, since $q \equiv 5$ $(\bmod 8)$, we get $2 \mid\left(1+q^{u}\right)$ and $4 \nmid\left(1+q^{u}\right)$. But then $1+q^{u}=2$ or 6 gives a contradiction and completes the proof.

\section{Proof of Density Theorem 2}

In order to prove the second density result, we would like to understand the solutions of the $S$-unit equation in the imaginary quadratic fields $K=\mathbb{Q}(\sqrt{-d})$ for squarefree $d$ such that $-d \equiv 1$ (mod 8). In this case, note that 2 splits in $K$ and $S=T=U=\left\{\mathfrak{P}_{1}, \mathfrak{P}_{2}\right\}$ where $2 \mathcal{O}_{K}=\mathfrak{P}_{1} \mathfrak{P}_{2}$. Suppose that $d>2$ and therefore the unit group of $\mathcal{O}_{K}$ is $\{ \pm 1\}$.

Lemma 8.1. Up to the action of $\mathfrak{S}_{3}$, every relevant element $(\lambda, \mu) \in \Lambda_{S}$ has the form

$$
\begin{array}{r}
\lambda=\frac{2^{r_{1}}-2^{r_{2}}+1+v \sqrt{-d}}{2}, \mu=\frac{2^{r_{2}}-2^{r_{1}}+1-v \sqrt{-d}}{2} \\
\text { where } r_{1} \geq r_{2} \geq 0, v \in \mathbb{Z}-\{0\} \\
\text { and satisfies } \quad\left(2^{r_{1}}-2^{r_{2}}+1\right)^{2}-2^{r_{1}+2}=-d v^{2} \\
\left(2^{r_{2}}-2^{r_{1}}+1\right)^{2}-2^{r_{2}+2}=-d v^{2}
\end{array}
$$

In fact, we obtain $r_{1}=r_{2}$ if $r_{1}, r_{2} \geq 3$, and equations 8.3 and 8.4 become $1-2^{r+2}=-d v^{2}$ where we set $r_{1}=r_{2}=r$. Also, we have the following exceptional cases: $r_{1}=1, r_{2}=0, r_{1}=2, r_{2}=1$, $r_{1}=3$ and $r_{2}=2$. Moreover, if $-d \equiv 1(\bmod 8)$ then $v$ is an odd integer. 
Proof. Assume that $\lambda$ and $\mu$ are given by equation 8.1 and satisfy the conditions of the lemma. Then, we see that $\lambda$ and $\mu$ are in $\mathcal{O}_{S}$ but not in $\mathbb{Q}^{*}$ and satisfy the $S$-unit equation $\lambda+\mu=1$. Furthermore, $\lambda$ and $\mu$ have norms $2^{r_{i}}$ by (8.3) and (8.4), and hence are in $\mathcal{O}_{S}^{*}$. Therefore, $(\lambda, \mu)$ is a relevant element of $\Lambda_{S}$.

Coversely, suppose that $(\lambda, \mu)$ is a relevant element of $\Lambda_{S}$. By Lemmas [5.1] and 7.1, we can assume that $\lambda, \mu \in \mathcal{O}_{K}-\mathbb{Q}$. Let $\bar{x}$ denotes the conjugation for any $x \in K$.

Now, $\lambda \bar{\lambda}=2^{r_{1}}$ and $\mu \bar{\mu}=2^{r_{2}}$ and we may assume $r_{1} \geq r_{2} \geq 0$. We note that

$$
\lambda+\bar{\lambda}=\lambda \bar{\lambda}-(1-\lambda)(1-\bar{\lambda})+1=\lambda \bar{\lambda}-\mu \bar{\mu}+1=2^{r_{1}}-2^{r_{2}}+1
$$

and $\lambda-\bar{\lambda}=v \sqrt{-d}$ where $v \in \mathbb{Z}-\{0\}$ since $\lambda \notin \mathbb{Q}$. By combining the previous expressions, we get the equation given in (8.1). The equations 8.3 and 8.4 follow from the identity $(\lambda+\bar{\lambda})^{2}-(\lambda-\bar{\lambda})^{2}=4 \lambda \bar{\lambda}$ and the corresponding identity for $\mu$.

Now, assume $r_{1}>r_{2} \geq 3$ and $r_{1}=r_{2}+a$ where $a \geq 1$. Then from the equations 8.3 and 8.4 , we have

$$
\left(2^{r_{2}+a}-2^{r_{2}}+1\right)^{2}<2^{r_{2}+a+2} \text { and }\left(2^{r_{2}}-2^{r_{2}+a}+1\right)^{2}<2^{r_{2}+2} .
$$

By expanding the squares and adding the two inequalities we get:

$$
2^{2 r_{2}+2 a+1}+2^{2 r_{2}+1}-2^{2 r_{2}+a+2}<2^{r_{2}+a+2}+2^{r_{2}+2}-2<2^{r_{2}+a+2}+2^{r_{2}+2} .
$$

In other words, we have

$$
2^{2 r_{2}+2 a+1}+2^{2 r_{2}+1}-2^{2 r_{2}+a+2}<2^{r_{2}+2}\left(2^{a}+1\right) .
$$

Now, after dividing both sides by $2^{r_{2}+2}$ and rearranging we get:

$$
2^{r_{2}-1}\left(2^{a}-1\right)^{2}<2^{a}+1 \text {. }
$$

Since $r_{2} \geq 3$, then $2^{\frac{r_{2}-1}{2}} \geq 2^{1}=2$ and $\sqrt{2^{a}+1}<2^{a}$ when $a \geq 1$. Hence, we obtain

$$
2\left(2^{a}-1\right)<2^{a} \rightarrow 2^{a}<2,
$$

which is possible only when $a=0$. This gives a contradiction and therefore we get $a=0$, i.e. $r_{1}=$ $r_{2} \geq 3$. Exceptional cases can be verified easily. Finally, if we reduce the equation $1-2^{r+2}=-d v^{2}$ modulo 8 , we get $1 \equiv v^{2}(\bmod 8)$ implying $v$ is a nonzero odd integer. Note that if $-d \equiv 5(\bmod 8)$ or $-d \equiv 2$ or $3(\bmod 4)$ there are no relevant solutions.

Now we will show that under the assumptions of Density Theorem 2, the solutions of the unit equation have the necessary assumptions to apply the Main Theorem i.e. a solution $(\lambda, \mu)$ satisfies $\max \left\{\left|v_{\mathfrak{P}}(\lambda)\right|,\left|v_{\mathfrak{P}}(\mu)\right|\right\} \leq 4 v_{\mathfrak{P}}(2)$ where $\mathfrak{P}$ is a prime lying over 2 with inertia degree 1 . This will complete the proof of Density Theorem 2.

Since $K=\mathbb{Q}(\sqrt{-d})$ and $-d \equiv 1(\bmod 8), 2$ splits in $\mathcal{O}_{K}$ and hence we need to verify that $\max \left\{\left|v_{\mathfrak{P}}(\lambda)\right|,\left|v_{\mathfrak{P}}(\mu)\right|\right\} \leq 4 v_{\mathfrak{P}}(2)$ for any solution $(\lambda, \mu)$ of the $S$-unit equuation $\lambda+\mu=1$. Note that it is enough to check this for any representative of an orbit under the action of $\mathfrak{S}_{3}$ by Lemma 6.2(i) of [4]. Assume that $\max \left\{\left|v_{\mathfrak{P}}(\lambda)\right|,\left|v_{\mathfrak{P}}(\mu)\right|\right\}=\left|v_{\mathfrak{P}}(\lambda)\right|=r>4$. By Lemma [8.1, $\lambda=\frac{1+v \sqrt{-d}}{2}$ where $1+d v^{2}=2^{r+2}$.

Consider $1+d v^{2}=2^{r+2}$ modulo 6 . Since $2^{r+2} \equiv 2$ or $4(\bmod 6)$ we get $d v^{2} \equiv 1$ or $3(\bmod 6)$. Using the assumption that $d \equiv 5(\bmod 6)$ and multiplying both sides with 5 we get $v^{2} \equiv 5$ or 3 $(\bmod 6)$. Since $v^{2} \equiv 5(\bmod 6)$ is not possible we get $v^{2} \equiv 3(\bmod 6)$ i.e. $v$ is divisible by 3 and $v^{3}$ 
is divisible by 9 . Now using this information and reducing the equation $1+d v^{2}=2^{r+2}$ modulo 9 we get $1 \equiv 2^{r+2}(\bmod 9)$ which implies that $6 \mid(r+2)$.

To finish the proof we consider $1+d v^{2}=2^{r+2}=2^{6 k}$ modulo 14 . Since $k>1$ we get $1+d v^{2}=$ $2^{r+2} \equiv 8(\bmod 14)$ i.e. $d v^{2} \equiv 1(\bmod 14) . \quad$ Using the fact that $v^{2} \equiv 0,1,2,4,8,9,11(\bmod 14)$ we get that the only solution is when $d \equiv 7(\bmod 14)$, which is a contradiction to the second assumption of Density Theorem 2.

\section{Proof of Density Theorem 3}

Our reference for the notation used in this section is [4]. We will rewrite it here for the convenience of the reader.

Let $\mathcal{U}$ be a set of positive integers and $X$ be a positive real number. Then, define $\mathcal{U}(X)=\{d \in$ $\mathcal{U}: d \leq X\}$. If the limit $\delta(\mathcal{U})=\lim _{X \rightarrow \infty} \frac{\# \mathcal{U}(X)}{X}$ exists, it is called the absolute density of $\mathcal{U}$.

Define $\mathbb{N}^{s f}=\{d \geq 2: d$ squarefree $\}$. If the limit

$$
\delta_{\text {rel }}(\mathcal{U})=\lim _{X \rightarrow \infty} \frac{\#\{d \in \mathcal{U}: d \leq X\}}{\#\left\{d \in \mathbb{N}^{s f}: d \leq X\right\}}=\lim _{X \rightarrow \infty} \frac{\# \mathcal{U}(X)}{\# \mathbb{N}^{s f}(X)}
$$

for a subset $\mathcal{U} \subset \mathbb{N}^{s f}$ exists, then it called the relative density of $\mathcal{U}$ in $\mathbb{N}^{s f}$.

Throughout this section, we will use the following two classical theorems from analytic number theory.

Theorem 9.1. (e.g [Lan09,p.636].) For integers $r$ and $N$ with $N$ positive, let $\mathbb{N}_{r, N}^{s f}=\left\{d \in \mathbb{N}^{s f}\right.$ : $d \equiv r(\bmod N)\}$. Let $s=\operatorname{gcd}(r, N)$ and suppose that $s$ is squarefree. Then

$$
\# \mathbb{N}_{r, N}^{s f}(X) \sim \frac{\varphi(N)}{s \varphi(N / s) N \prod_{q \mid N}\left(1-q^{-2}\right)} \frac{6}{\pi^{2}} X
$$

where $\varphi$ denotes the Euler's totient function.

Theorem 9.2. (e.g. [Lan09,pp.641-643]). Let $N$ be a positive integer. Let $r_{1}, \ldots, r_{m}$ be distinct modulo $N$, satisfying $\operatorname{gcd}\left(r_{i}, N\right)=1$. Let $E$ be the set of positive integers $d$ such that every prime factor $q$ of $d$ satisfies $q \equiv r_{i}(\bmod N)$ for some $i=1, \ldots k$. Then there is some positive constant $\gamma=\gamma\left(N, r_{1}, \ldots, r_{m}\right)$ such that

$$
\# E(X) \sim \gamma \frac{X}{\log (X)^{1-m / \varphi(N)}}
$$

Remark 9.3. Observe that if we apply Theorem 9.1 to $\mathbb{N}^{s f}=\mathbb{N}_{0,1}^{s f}$, we obtain $\# \mathbb{N}^{s f}(X) \sim 6 X / \pi^{2}$. Moreover, for a subset $\mathcal{U}$ of $\mathbb{N}^{s f}, \delta(\mathcal{U})$ exists if and only if $\delta_{\text {rel }}(\mathcal{U})$ exists and $\delta_{\text {rel }}(\mathcal{U})=\pi^{2} \delta(\mathcal{U}) / 6$.

We would like to prove the following theorem.

Theorem 9.4. Let $\mathcal{C}$ be the set of $d \in \mathbb{N}^{s f}$ such that the $S$-unit equation 1.2 has no relevant solutions in $\mathbb{Q}(\sqrt{-d})$ and $\mathcal{D}=\{d \in \mathcal{C}:-d \not \equiv 5(\bmod 8)\}$. Then $\delta_{\text {rel }}(\mathcal{C})=1$ and $\delta_{\text {rel }}(\mathcal{D})=5 / 6$.

In order to prove Theorem 9.4, we need the following lemma.

Lemma 9.5. Let $\mathcal{C}^{\prime}=\mathbb{N}^{s f} \backslash \mathcal{C}$. Then $\delta\left(\mathcal{C}^{\prime}\right)=0$.

By assuming Lemma 9.5, we can prove Theorem 9.4 as below.

Proof of Theorem 9.4: By Lemma 9.5 and Remark 9.3, we have $\delta_{\text {rel }}\left(\mathcal{C}^{\prime}\right)=\delta\left(\mathcal{C}^{\prime}\right)=0$. The fact that $\mathcal{C}$ and $\mathcal{C}^{\prime}$ are complements in $\mathbb{N}^{s f}$ yields $\delta_{\text {rel }}(\mathcal{C})=1$.

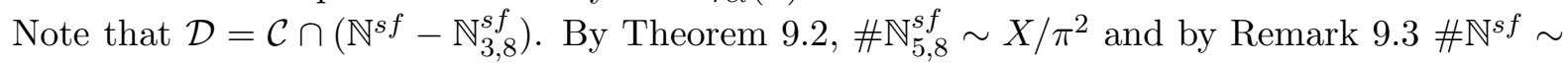
$6 X / \pi^{2}$. Hence, $\delta_{\text {rel }}(\mathcal{D})=5 / 6$. 


\subsection{Proof of Lemma 9.5}

In this section, we give the proof of Lemma 9.5. Throughout this section, we follow the proof of Lemma 7.1 of [4]. As we deal with the imaginary quadratic fields, we use a simplified version of their argument.

Being the complement of $\mathcal{C}$, the set $\mathcal{C}^{\prime}$ consists of squarefree $d \geq 2$ such that the $S$-unit equation 1.2 has a relevant solution in $\mathbb{Q}(\sqrt{-d})$. By Lemma 8.1, this set exactly contains squarefree $d \geq 2$ satisfying $1-2^{r+2}=-d v^{2}$ where $r \geq 3$ and $v$ is an odd integer. Now, for $\kappa_{1}=0$ and $\kappa_{2}=1$, we define $\mathcal{C}^{\prime}\left(\kappa_{1}\right)$ and $\mathcal{C}^{\prime}\left(\kappa_{2}\right)$ as the sets containing squarefree $d \geq 2$ satisfying $1-2^{r+2}=-d v^{2}$ where $r \geq 3$ and $v$ is an odd integer with $r \equiv \kappa_{1}$ and $\kappa_{2}(\bmod 2)$ respectively. Hence, $\mathcal{C}^{\prime}=\mathcal{C}^{\prime}\left(\kappa_{1}\right) \cup \mathcal{C}^{\prime}\left(\kappa_{2}\right)$. Assume $q$ is an odd divisor of $d$ and let $d \in \mathcal{C}^{\prime}\left(\kappa_{2}\right)$. Then reducing $1-2^{r+2}$ modulo $q$ implies that 2 is a quadratic residue modulo $q$. In this case, $q$ is an element of a proper subset of the congruence classes $\{\overline{1}, \overline{3}, \overline{5}, \overline{7}\}$ modulo 8 . Now by using Theorem 9.2 (note that in this case, the power of the logarithm in the theorem becomes less than 1$)$, we see that $\delta\left(\mathcal{C}^{\prime}\left(\kappa_{2}\right)\right)=0$

Now we will show that $\delta\left(\mathcal{C}^{\prime}\left(\kappa_{1}\right)\right)=0$. Assume $d \in \mathcal{C}^{\prime}\left(\kappa_{1}\right)$ and set $r=2 s, s \geq 2$. The condition for the relevant solutions becomes: $\left(2^{s+1}+1\right)\left(2^{s+1}-1\right)=d v^{2}$.

Let $\alpha_{1, s}=2^{s+1}+1$ and $\alpha_{2, s}=2^{s+1}-1$. Obviously, $\alpha_{1, s}$ and $\alpha_{2, s}$ are odd and coprime integers. Therefore, we can write $\alpha_{i, s}=d_{i, s} v_{i, s}^{2}$ where $d_{i, s}$ are squarefree and $d=\prod d_{i, s}$. Hence,

$$
\mathcal{C}^{\prime}\left(\kappa_{1}\right)=\left\{d_{1, s} d_{2, s}: s \geq 2\right\}
$$

Instead showing $\delta\left(\mathcal{C}^{\prime}\left(\kappa_{1}\right)\right)=0$, we will prove the equivalent statement that

$$
\delta_{\text {sup }}\left(\mathcal{C}^{\prime}\left(\kappa_{1}\right)\right)=\limsup _{X \rightarrow \infty} \# \mathcal{C}^{\prime}\left(\kappa_{1}\right)(X) / X=0 .
$$

The $m^{\text {th }}$ Mersenne number $M_{m}$ is defined to be $M_{m}=2^{m}-1$ for any positive integer $m$. Note that if $n \mid m$, then $M_{n} \mid M_{m}$.

Lemma 9.6. Let $m$ be a positive integer. Let $M_{m}=2^{m}-1$. Assume that $s_{1} \equiv s_{2}(\bmod m)$. Then $\alpha_{1, s} \equiv \alpha_{2, s}\left(\bmod M_{m}\right)$ for $i=1,2$.

Proof. Assume without loss of generality that $s_{1}>s_{2}$. Since $s_{1} \equiv s_{2}(\bmod m), m \mid\left(s_{1}-s_{2}\right)$. Then, $\alpha_{i, s_{1}}-\alpha_{i, s_{2}}=2^{s_{1}+1}-2^{s_{2}+1}=2^{s_{2}+1}\left(2^{s_{1}-s_{2}}-1\right)$. Since $m \mid\left(s_{1}-s_{2}\right)$ we get $M_{m} \mid\left(\alpha_{i, s_{1}}-\alpha_{i, s_{2}}\right)$.

Lemma 9.7. Let $m$ be a positive integer and $s_{0} \geq 2$ and $\mathcal{A}_{m, s_{0}}=\left\{d_{1, s}: s \equiv s_{0}(\bmod m)\right\}$. Let $p_{1}, \ldots, p_{k}$ be distict primes dividing $M_{m}$ that do not divide $\alpha_{1, s_{0}}$, and write $N=p_{1} \ldots p_{k}$. Then

$$
\# \mathcal{A}_{m, s_{0}}(X) \leq 2^{-k} X+N
$$

Proof. Assume $s \equiv s_{0}(\bmod m)$. Since $N \mid M_{n}, \alpha_{1, s} \equiv \alpha_{1, s_{0}}$ by Lemma 9.6. Moreover, $\alpha_{1, s}$ is coprime to $N$ as $\alpha_{1, s_{0}}$ is coprime to $N$. Hence, $v_{1, s}^{2}$ is coprime to $N$ where $\alpha_{i, s}=d_{i, s} v_{i, s}^{2}$. By reducing modulo $N$ we obtain $d_{1, s} \equiv \alpha_{1, s_{0}} v_{1, s}^{-2}(\bmod N)$, i.e. $d_{1, s} \in\left\{\alpha_{1, s_{0}} \omega^{2} \mid \omega \in(\mathbb{Z} / N \mathbb{Z})^{*}\right\}$. This set has cardinality $\varphi(N) / 2^{k}$ since $N$ is squarefree with $k$ distinct prime factors. Then the lemma follows.

Let us denote by $\omega(n)$ the number of distinct prime divisors of a positive integer $n$.

Lemma 9.8. For $m \geq 1$, let $h_{m}=\omega\left(M_{m}\right)$. Then $\delta_{\text {sup }}\left(\mathcal{C}^{\prime}\left(\kappa_{1}\right)\right) \leq 2^{-h_{m} / 2} m$. 
Proof. Let $k$ and $N$ as in Lemma 9.7 for some fixed $s_{0}$. Let

$$
\mathcal{C}^{\prime}\left(\kappa_{1}\right)_{m, s_{0}}=\left\{d_{1, s} d_{2, s}: s \geq 2, s \equiv s_{0} \quad(\bmod m)\right\} .
$$

Then, clearly

$$
\# \mathcal{C}^{\prime}\left(\kappa_{1}\right)_{m, s_{0}}(X) \leq \# \mathcal{A}_{\left.m, s_{0}\right)}(X) \leq 2^{-k} X+N \leq 2^{-k} X+M_{m}
$$

Let $q_{1}, \ldots, q_{k}^{\prime}$ be the distinct primes dividing $M_{m}$ that do not divide $\alpha_{2, s_{0}}$. A similar argument to Lemma 9.7 gives

$$
\# \mathcal{C}^{\prime}\left(\kappa_{1}\right)_{m, s_{0}}(X) \leq 2^{-k^{\prime}} X+M_{m} .
$$

Note that $\alpha_{1, s_{0}}$ and $\alpha_{2, s_{0}}$ are coprime implying that either $k \geq h_{m} / 2$ or $k^{\prime} \geq h_{m} / 2$. Therefore,

$$
\# \mathcal{C}^{\prime}\left(\kappa_{1}\right)_{m, s_{0}}(X) \leq 2^{-h_{m} / 2} X+M_{m} .
$$

If we set $s_{1}, \ldots, s_{m}$ be a complete system of representatives for $s$ modulo $m$, then

$$
\# \mathcal{C}^{\prime}\left(\kappa_{1}\right)(X) \leq 2^{-h_{m} / 2} X m+m M_{m} .
$$

Lemma 9.9. With notation as above, $\delta\left(\mathcal{C}^{\prime}\left(\kappa_{1}\right)\right)=0$.

Proof. By Lemma 9.8, we have

$$
0 \leq \delta_{\text {sup }}\left(\mathcal{C}^{\prime}\left(\kappa_{1}\right)\right) \leq \frac{m}{2^{\left(h_{m} / 2\right)}} \leq \frac{m}{2^{\left(2^{\omega(m)-1}\right)-1}}
$$

for any $m \geq 1$, where the last inequality follows from the fact that $h_{m} \geq 2^{\omega m}-2$ [Corollary 7.5 in [4]]. Assuming $y$ is large and setting $m=\prod_{p \leq y} p$, note that $\omega(m)=\pi(y)$ and $m=\exp (\vartheta(y))$, where $\pi$ and $\vartheta$ are the prime counting function and the first Chebyshev function. Since

$$
\pi(y) \sim y / \log y \text { and } \vartheta(y) \sim y
$$

by the prime number theorem (see e.g., [Apo76, ch. 4]), we obtain $\delta_{\text {sup }}\left(\mathcal{C}^{\prime}\left(\kappa_{1}\right)\right)=0$ as $y \rightarrow \infty$ in 9.1

\section{References}

[1] G. Cornell, J. H. Silverman, G. Stevens and [et al.], Modular forms and Fermat's last theorem, (Springer-Verlag, New York, 1997). 3

[2] T. M. Apostol, Introduction to analytic number theory, (Springer, New York, 1976).

[3] H. Deconinck, On the generalized Fermat equation over totally real fields, Acta Arith. 173 (2016), no. 3, $225-237$. 1, 5, $5,7,7$

[4] N. Freitas and S. Siksek, The asymptotic Fermat's last theorem for five-sixths of real quadratic fields, Compos. Math. 18 (2015), no. 8, 1395-1415. 1, 6, 7, 7, 8, 9, 9.1, 9.1

[5] E. Landau, Handbuch der Lehre von der Verteilung der Primzahlen II, (B.G.Teubner,Leipzig,1909).

[6] J-P. Serre, Sur les représentations modulaires de degré 2 de $\operatorname{Gal}((\bar{Q}) / \mathbb{Q})$, Duke Math. J. $54(1987)$, 179-230. 3

[7] J. H. Silverman, The arithmetic of elliptic curves, Graduate Texts in Mathematics, vol. 106 (Springer, Dordrecht, 1986). 5

[8] M. H. Şengün and S. Siksek, On the asymptotic Fermat's last theorem over number fields, Comment. Math. Helv. 93 (2018), no. 2, 359-375. 1, 1 , 2, 2.1, 2.1 2.2, 3, 4.1, 4.2, 4.2

Yasemin Kara, Bogazici University, Faculty of Arts and Sciences, Mathematics Department, Bebek, IstANBUL, 34342, TURKEY

E-mail address, Yasemin Kara: yasemin.kara@boun.edu.tr

Ekin Ozman, Bogazici University, Faculty of Arts and Sciences, Mathematics Department, Bebek, Istanbul, 34342, TURKeY

E-mail address, Ekin Ozman: ekin.ozman@boun.edu.tr 